\title{
Felder von Flächenelementen in 4-dimensionalen Mannigfaltigkeiten
}

\author{
Von \\ Friedrich Hirzebruch in Bonn und Heinz HopF in Zürich
}

Herrn H. BehNk zum 60. Geburtstag gewidmet

\section{Einleitung}

Wir betrachten 4-dimensionale kompakte orientierte differenzierbare Mannigfaltigkeiten $M$ und in ihnen stetige Felder von orientierten Flächenelementen. Die nächstliegende Frage lautet: ,In welchen $M$ existieren solche Felder ohne Singularitäten?" Eines der Hauptergebnisse dieser Arbeit besteht darin, daß diese $M$ durch eine arithmetische Relation zwischen der Eulerschen. Charakteristik $e$ und der Poincaréschen Bilinearform $S(x, y)$ charakterisiert werden; $S(x, y)$ bezeichnet also die Schnittzahl der 2-dimensionalen Homologieklassen $x$ und $y$, und zwar ist "Homologie" hier im einfachsten Sinne, nämlich als "Homologie mit Division" zu verstehen $(x, y$ sind also Elemente der, modulo der Torsionsgruppe reduzierten, ganzzahligen Homologiegruppe). Ubrigens werden wir uns der Cohomologie-Sprache bedienen, so daß nachher $x, y$ Elemente der 2-dimensionalen, modulo der Untergruppe der Elemente endlicher Ordnung reduzierten, ganzzahligen Cohomologiegruppe und $S(x, y)$ den Wert des 4-dimensionalen Cup-Produktes $x y$ auf dem Grundzyklus der orientierten $M$ bezeichnen werden. In der erwähnten arithmetischen Relation werden außer dem Trägheitsindex (oder der Signatur) $\tau$ (vgl. 4.1) einer symmetrischen Bilinearform $S(x, y)$ noch die folgenden Begriffe auftreten:

Auf dem $p$-dimensionalen Gitter (ganzzahligen Modul) $H$ sei $S$ eine ganzzahlige symmetrische bilineare Funktion von Gittervektoren $x, y$ mit der Determinante \pm 1. Dann gibt es solche Gittervektoren $w$, daß

$$
S(w, x) \equiv S(x, x) \bmod 2 \text { für jeden } x \in H
$$

ist; in der Tat: hat $S$ in bezug auf eine beliebig gewählte Basis von $H$ die Matrix $\left(a_{i j}\right)$, so besitzt der Vektor $w$, dessen Komponenten $w_{j}$ durch das Gleichungssystem

$$
\sum_{j=1}^{p} a_{i j} w_{j}=a_{i i}, \quad i=1,2, \ldots, p,
$$

bestimmt sind, die Eigenschaft (1); ferner sieht man leicht, daß mit $w$ alle Vektoren $w^{\prime}=w+2 x\left(x \in H\right.$ beliebig), und nur diese $w^{\prime}$, ebenfalls (1) erfüllen. Somit bilden die durch (1) charakterisierten $w$ eine Restklasse $W \in H / 2 H$. 
Wir verstehen unter $\Omega$ die Menge aller Zahlen $S(w, w)$ mit $w \in W$. (Im Falle $p=0$ verstehen wir unter $Q$ die nur aus der 0 bestehende Menge.)

Für eine Mannigfaltigkeit $M$ ist also neben der Eulersohen Charakteristik $e$ und dem Trägheitsindex $\tau$ die Zahlenmenge $\Omega$ definiert. Nun lautet die Antwort auf die anfangs formulierte Frage:

In $M$ gibt es dann und nur dann Felder von Flächenelementen ohne Singularität, wenn

$$
3 \tau+2 e \in \Omega \text { und } 3 \tau-2 e \in \Omega .
$$

Felder mit höchstens endlich vielen Singularitäten existieren nach einem Satz von WHITNEY (den wir übrigens in (4.1 vi) noch einmal beweisen werden) in jeder $M$. Jeder isolierten Singularität $P_{i}$ ist in bekannter Weise ein Index zugeordnet: er ist die Homotopieklasse einer bestimmten Abbildung einer Sphäre $\mathbf{S}_{\mathbf{3}}$ in die Mannigfaltigkeit $\Sigma$ der orientierten Ebenen durch einen Punkt des euklidischen $\mathbf{R}^{4}$, also ein Element der Gruppe $\pi_{3}(\Sigma)$; da $\Sigma$ homöomorph mit dem Sphärenprodukt $\mathbf{S}_{\mathbf{2}} \times \mathbf{S}_{\mathbf{2}}$ und da $\pi_{3}\left(\mathbf{S}_{2}\right)$ unendlich zyklisch ist, kanh der Index bei Beachtung gewisser Orientierungskonventionen als Paar $\left(a_{i}, b_{i}\right)$ ganzer Zahlen aufgefaßt werden; die Indexsumme des Feldes ist das Paar $(a, b)$ mit $a=\Sigma a_{i}, b=\Sigma b_{i}$, summiert über alle Singularitäten. Wir fragen, welche Indexsummen in einer gegebenen $M$ auftreten. Diese Indexsummen sind im allgemeinen nicht einander gleich; vielmehr gilt folgender Satz:

Dann und nur dann haben in $M$ alle Indexsummen den gleichen Wert, wenn die zweite Bettische Zahl $b_{2}=0$ ist; und zwar ist dieser Wert

$$
(a, b)=(-e / 2,+e / 2)=\left(-1+b_{1}, 1-b_{1}\right),
$$

wobei $b_{1}$ die erste Bettische Zahl ist. Wenn $b_{2} \neq 0$ ist, so gibt es in $M$ Felder mit unendlich vielen verschiedenen Indexsummen.

Dies ist ein Korollar des folgenden Hauptsatzes der Arbeit:

Als Indexsummen von Feldern mit endlich vielen Singularitäten in $M$ treten die folgenden Zahlenpaare $(a, b)$ und nur diese auf:

$$
\left.a=\frac{1}{4}(\alpha-3 \tau-2 e), \quad b=\frac{1}{4}(\beta-3 \tau+2 e) \text { mit beliebigen } \alpha, \beta \in \Omega{ }^{1}\right) .
$$

1) Da $a$ und $b$ nach Definition ganze Zahlen sind, sind auch die rechten Seiten in (2), die man als

$$
\frac{1}{4}(\alpha-\tau)-\frac{1}{2}(\tau+e), \quad \frac{1}{4}(\beta-\tau)-\frac{1}{2}(\tau-e)
$$

sohreiben kann, ganz; ferner ist $\tau \equiv e$ mod. 2 (da beide Zahlen mod. 2 der 2. Bettischen Zahl kongruent sind); daher ist die Ganzheit der obigen Zahlen gleichbedeutend mit der Gültigkeit der Kongruenzen

$$
S(w, w) \equiv \tau \bmod .4 \text { für } w \in W .
$$

Für diejenigen Bilinearformen $\boldsymbol{S}$, welche als Poincarésche Formen in Mannigfaltigkeiten $\boldsymbol{M}$ auftreten, haben wir somit (3) auf dem Umweg über (2) bewiesen. Herr W. LedermanN hat auf algebraischem. Wege gezeigt, daB (3) für alle symmetrischen ganzzahligen Bilinearformen $\mathbb{S}$ mit ungerader Determinante gilt (An arithmetical property of quadratic forms, Comment. Math. Helvet. Erscheint demnächst). 
Wenn wir ein festes $w \in W$ auszeichnen und

$$
w^{2}-3 \tau-2 e=4 a_{0}, w^{2}-3 \tau+2 e=4 b_{0}
$$

setzen, so können wir statt (2) auch schreiben:

$$
a=x^{2}+w x+a_{0}, b=y^{2}+w y+b_{0} \text { mit beliebigen } x, y \in H
$$

(wobei $H$ die, modulo der Cotorsion reduzierte, ganzzahlige 2. Cohomologiegruppe ist und hier ein Produkt $u v$ zweier Elemente $u, v \in H$-also z. B. $x^{2}$, $w x, \ldots$ - ebenso wie $S(u, v)$ den Wert des 4-dimensionalen Cup-Produktes auf dem Grundzyklus von $M$ bezeichnet).

2-Beine, d. h. geordnete Paare linear unabhängiger Richtungen, stellen Flächenelemente dar. Es gilt:

In $M$ gibt es dann und nur dann Felder von 2-Beinen ohne Singularität (d.h. Paare von überall linear unabhängigen Richtungsfeldern), wenn $e=0$ und $3 \tau \in \Omega$ ist.

Auch die möglichen Indexsummen der Singularitäten von 2-Bein-Feldern werden bestimmt. Ferner werden Kriterien für die Existenz einer fastkomplexen Struktur sowie für die Parallelisierbarkeit von $M$ angegeben. Alle Ergebnisse sind in $(4.3-4.6)$ zusammengestellt.

DaB die Indexsummen $(a, b)$ dureh die Werte zweier Cohomologie-Polynome 2. Grades von der Art (2') bestimmt sind, war bereits - übrigens auf einem anderen Wege als dem, den wir jetzt benutzen werden - in den Noten [8] und [9] von Horf festgestellt worden; jedoch gelang damals die Bestimmung der Koeffizienten dieser Polynome nur in Spezialfällen, z. B. für die komplexe projektive Ebene, aber nieht für beliebige $M$. Der allgemeine Fall wurde dann von HIRzEBRUCH erledigt. Die ganze Untersuchung betrifft natürlich Faserbündel, Hindernisse und charakteristische Klassen. Wir werden in unserer Darstellung Sätze aus diesen Theorien ohne Kommentar benutzen; wir verweisen auf [12] (besonders für die Hindernis-Theorie) und auch auf $[1,2,3,6]$ (für Faserbündel und charakteristische Klassen). Die Tatsache, daB unsere Sätze, zu denen wir auf erst in den letzten Jahren erschlossenen Wegen gelangen, ganz im Rahmen der alten Poincaréschen Begriffe formuliert werden können (wie wir es oben getan haben), hat vielleicht keine besonders große prinzipielle Bedeutung (da sie sich kaum auf höhere Dimensionszahlen übertragen lassen dürfte); aber wir halten sie doch für bemerkenswert nicht nur als Kuriosum, sondern auch im Hinblick auf die Beziehungen zwischen alten Begriffen und neuen Methoden, die sich in ihr äußern.

Aus Gründen teils sachlicher, teils persönlicher Natur, die wir angedeutet. haben, macht es uns besondere Freude, diese Arbeit Herrn BeHrke zu widmen, dessen Interessen und dessen Wirksamkeit in so hohem Maße der gegenseitigen Durchdringung der „klassischen" und der "modernen" Mathematik sowie der Zusammenarbeit zwischen Mathematikern verschiedener Generationen gelten. 


\section{§1. Homomorphismen und charakteristische Klassen}

1.1. Wie in [2] werden Faserbündel durch ein Symbol, meistens durch einen kleinen griechischen Buchstaben, angedeutet. Ist $\xi$ ein Faserbündel, dann wird mit $E_{\xi}$ der Totalraum und mit $B_{\xi}$ die Basis von $\xi$ bezeichnet. Es sei $G$ eine kompakte Liesche Gruppe und $\xi$ ein $G$-Prinzipalfaserbündel. Wenn $G$ auf dem Raum $F$ operiert, dann hat man bekanntlich das zu $\xi$ assoziierte Faserbündel $(\xi, F)$ mit $F$ als Faser, dessen Totalraum $E_{\xi} \times{ }_{G} F$ ist, das ist der Quotient von $E_{\xi} \times F$ modulo der Äquivalenzrelation $(x, f) \approx$ $\approx\left(x \cdot g, g^{-1} \cdot f\right)$. Es sei $G^{\prime}$ eine weitere kompakte Liesche Gruppe und $\lambda$ ein Homomorphismus von $G$ in $G^{\prime}$. Jedem $G$-Prinzipalfaserbündel $\xi$ ist dann die $\lambda$-Erweiterung $\lambda_{*} \xi$ zugeordnet, das ist ein $G^{\prime}$-Prinzipalfaserbündel, ebenfalls mit der Basis $B_{\xi}$, dessen Totalraum $E_{\xi} \times{ }_{G} G^{\prime}$ ist, wo $G$ auf $G^{\prime}$ durch $g \cdot g^{\prime}=$ $=\lambda(g) \cdot g^{\prime}$ operiert. Operiert $G^{\prime}$ auf $F$, dann operiert auch $G$ auf $F$ durch $g \cdot f=\lambda(g) \cdot f$ und der Totalraum von $\left(\lambda_{*} \xi, f\right)$ ist kanonisch homöomorph zum Totalraum von $(\xi, F)$ :

$$
\left(E_{\xi} \times{ }_{G} G^{\prime}\right) \times{ }_{G^{\prime}} F^{\prime} \cong E_{\xi} \times{ }_{G} F .
$$

Dieser Homöomorphismus ist fasertreu. Weiter werde an folgendes erinnert: Wenn $\xi$ ein $G$-Prinzipalfaserbündel und $U$ eine abgeschlossene Untergruppe von $G$ ist, dann ist $E_{\xi} / U$ der Totalraum des zu $\xi$ assoziierten Faserbündels mit $G / U$ als Faser, wo $G$ auf $G / U$ durch Linkstranslationen operiert.

$$
E_{\xi} / U=E_{\xi} \times{ }_{G} G / U \text {. }
$$

Dieses $G / U$-Faserbündel hat dann und nur dann einen Schnitt über $B_{\xi}$, wenn $\xi$ die $\iota$-Erweiterung eines $U$-Prinzipalfaserbündels über $B_{\xi}$ ist, wo $\iota$ die Einbettung von $U$ in $G$ ist. - In [2] wird ein Verfahren zur Berechnung der charakteristischen Klassen von $\lambda_{*} \xi$ aus denen von $\xi$ angegeben, das wir auf Homomorphismen von $\mathbf{S O}(4)$ in $\mathbf{S O}(3)$ und von $\mathbf{U}_{(2)}$ in $\mathbf{S O}(3)$ anwenden werden. Wie üblich ist $\mathbf{S O}(k)$ die Gruppe der orthogonalen $\mathbf{A b b i l d u n g e n ~ d e s ~} \mathbf{R}^{k}$. mit der Determinante 1 und $\mathbf{U}(k)$ die unitäre Gruppe im $\mathbf{C}^{k}$. Die in dieser Arbeit auftretenden G-Prinzipalfaserbündel haben folgende charakteristische Klassen:

i) $G=\mathbf{S O}(4)$. Man hat die Stiefel-Whitneyschen Klassen $w_{2}(\xi) \epsilon$ $H^{2}\left(B_{\xi}, \mathbf{Z}_{2}\right)$ und $W_{3}(\xi) \in H^{3}\left(B_{\xi}, \mathbf{Z}\right)$, ferner die Eulersche Klasse $W_{4}(\xi)$ $\in H^{4}\left(B_{\xi}, \mathbf{Z}\right)$ und die Pontrjaginsche Klasse $p_{1}(\xi) \in H^{4}\left(B_{\xi}, \mathbf{Z}\right)$.

ii) $G=\mathbf{S O}(3)$. Man hat die Stiefel-Whitneyschen Klassen $w_{\mathbf{2}}(\xi)$ $\in H^{2}\left(B_{\xi}, \mathbf{Z}_{2}\right)$ und $W_{3}(\xi) \in H^{3}\left(B_{\xi}, \mathbf{Z}\right)$, ferner die Pontrjaginsche Klasse $p_{1}(\xi)$ $\in H^{4}\left(B_{\xi}, \mathbf{Z}\right)$.

iii) $G=\mathbf{U}(n)$. Man hat die Chernschen Klassen $c_{i}(\xi) \in H^{2 i}\left(B_{\xi}, \mathbf{Z}\right)$, $(i=1,2, \ldots, n)$.

In i) und ii) ist $W_{3}=\delta_{*} w_{2}$, wo $\delta_{*}$ der zur Koeffizientensequenz $0 \rightarrow \mathbf{Z}$ $\stackrel{2}{\rightarrow} \mathbf{Z} \rightarrow \mathbf{Z}_{2} \rightarrow 0$ gehörige Homomorphismus $H^{2}\left(B_{\xi}, \mathbf{Z}_{2}\right) \rightarrow H^{3}\left(B_{\xi}, \mathbf{Z}\right)$ ist. Also ist $2 W_{3}=0$. Die Stiefel-Whitneyschen, die Eulerschen und die Chernschen Klassen können bekanntlich als erste Hindernisse gewisser assoziierter Faserbündel definiert werden [12]. Die Pontrjaginsche Klasse $p_{1}(\xi)$ ist gleich 
$-c_{2}\left(\lambda_{*} \xi\right)$, wo $\lambda$ hier die ,komplexe Erweiterung" $\mathbf{S O}(4) \rightarrow \mathbf{U}(4)$ bzw. $\mathbf{S O}(3) \rightarrow \mathbf{U}(3)$ ist.

1.2. Die Homomorphismen $\lambda^{(1)}, \lambda^{(2)}$ von $\mathbf{S O}(4)$ auf SO (3). Der Vektorraum $\mathbf{R}^{4}$ wird mit dem Körper $\mathbf{K}$ der Quaternionen identifiziert:

$$
x=\left(x_{1}, x_{2}, x_{3}, x_{4}\right)=x_{1}+i x_{2}+j x_{3}+k x_{4}=x_{1}+i x_{2}+\left(x_{3}+i x_{4}\right) \cdot j
$$

Der komplexe Körper $\mathbf{C}$ ist in $\mathbf{K}$ enthalten $\left(x_{3}=x_{4}=0\right)$. Mit $\mathbf{S}_{3}$ bezeichnen wir die multiplikative Gruppe der Quaternionen vom Betrage 1 und mit $\mathbf{S}_{\mathbf{1}}$ die multiplikative Gruppe der komplexen Zahlen vom Betrage 1. Es ist also $\mathbf{S}_{1}=\mathbf{S}_{3} \cap \mathbf{C}$. Das Element $\left(q_{1}, q_{2}\right)$ der Gruppe $\mathbf{S}_{3} \times \mathbf{S}_{3}$ operiert auf $\mathbf{R}^{4}(=\mathbf{K})$ dureh

$$
\left(q_{1}, q_{2}\right)(x)=q_{1} \cdot x \cdot q_{2}^{-1}, \quad(x \in \mathbf{K}, \text { Multiplikation im Sinne von } \mathbf{K}) .
$$

Man erhält einen Homomorphismus $\alpha$ von $\mathbf{S}_{3} \times \mathbf{S}_{3}$ auf $\mathbf{S O}(4)$, dessen Kern von $(-1,-1)$ erzeugt wird, und damit eine exakte Sequenz

$$
0 \rightarrow \mathbf{Z}_{\mathbf{2}} \rightarrow \mathbf{S}_{3} \times \mathbf{S}_{\mathbf{3}} \stackrel{\alpha}{\rightarrow} \mathbf{S O}(4) \rightarrow 0 \text {. }
$$

Der Vektorraum $\mathbf{R}^{\mathbf{3}}$ wird mit dem Raum der Quaternionen mit verschwindendem Realteil $\left(x_{1}=0\right)$ identifiziert. $q \in \mathbf{S}_{3}$ operiert auf $\mathbf{R}^{3}$ durch

$$
q(y)=q \cdot y \cdot q^{-1},
$$

Man erhält einen Homomorphismus $\beta$ von $\mathbf{S}_{3}$ auf SO (3), dessen Kern von -1 erzeugt wird, und damit eine exakte Sequenz

$$
0 \rightarrow \mathbf{Z}_{2} \rightarrow \mathbf{S}_{3} \stackrel{\beta}{\longrightarrow} \mathrm{SO}(3) \rightarrow 0 \text {. }
$$

Es sei $\Delta$ die Diagonale von $\mathbf{S}_{3} \times \mathbf{S}_{3}$. Dann ist $\alpha(\Delta)=1 \times \mathbf{S O}(3) \subset \mathbf{S O}(4)$. Mit $\pi_{r}(r=1,2)$ bezeichnen wir die Projektion von $\mathbf{S}_{3} \times \mathbf{S}_{3}$ auf seinen $r$-ten Faktor. Dann gibt es einen und nur einen Homomorphismus $\lambda^{(r)}$, so daß das folgende Diagramm kommutativ ist

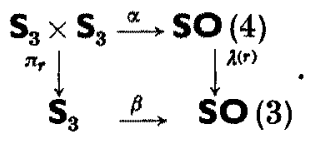

In den vier Gruppen dieses Diagramms zeichnen wir maximale Tori aus: In $\mathbf{S}_{3}$ die Untergruppe $\mathbf{S}_{\mathbf{1}}$ der Elemente $\exp (2 \pi i \varphi),(\varphi \in \mathbf{R})$; in $\mathbf{S}_{\mathbf{3}} \times \mathbf{S}_{\mathbf{3}}$ die Untergruppe $\mathbf{S}_{1} \times \mathbf{S}_{1}$ der Elemente $\left(\exp \left(2 \pi i \varphi_{1}\right), \exp \left(2 \pi i \varphi_{2}\right)\right),\left(\varphi_{1}, \varphi_{2} \in \mathbf{R}\right)$; in SO (4) die Untergruppe SO (2) $\times$ SO (2) der Matrizen

wo

$$
\left(\begin{array}{lc}
D\left(t_{1}\right) & 0 \\
0 & D\left(t_{2}\right)
\end{array}\right), \quad\left(t_{1}, t_{2} \in \mathbf{R}\right)
$$

$$
D(t)=\left(\begin{array}{rr}
\cos 2 \pi t & -\sin 2 \pi t \\
\sin 2 \pi t & \cos 2 \pi t
\end{array}\right) ;
$$

schließlich in SO(3) die Untergruppe $1 \times \mathbf{S O}(2)$ der Matrizen

$$
\left(\begin{array}{cc}
1 & 0 \\
0 & D(u)
\end{array}\right), \quad(u \in \mathbf{R})
$$


Man kontrolliert sofort, daß dureh $\alpha, \beta, \pi_{r}$ im Diagramm (4) der ausgezeichnete maximale Torus auf den ausgezeichneten maximalen Torus der Bildgruppe abgebildet wird und da $B$ man die Abbildungen auf dem jeweiligen maximalen Torus durch folgendes Diagramm beschreiben kann

$$
\begin{aligned}
& \left(\varphi_{1}, \varphi_{2}\right) \rightarrow\left(t_{1}, t_{2}\right)=\left(\varphi_{1}-\varphi_{2}, \varphi_{1}+\varphi_{2}\right) \\
& \downarrow \\
& \varphi=\varphi_{r} \rightarrow u=2 \varphi .
\end{aligned}
$$

Damit wird die Abbildung $\lambda^{(1)}$ auf dem maximalen Torus von $\mathbf{S O}(4)$ durch $u=t_{1}+t_{2}$ gegeben und $\lambda^{(2)}$ durch $u=t_{2}-t_{1}$.

1.3. Der Homomorphismus $\mu$ von $\mathbf{U}(2)$ auf $\mathbf{S O}(3)$. Jedes Quaternion $x$ kann auf genau eine Weise in der Form

$$
x=z_{1}+z_{2} \cdot j,
$$

geschrieben werden (1). Dadurch wird $\mathbf{K}$ mit dem $\mathbf{C}^{2}$ identifiziert. Die komplexe Struktur in dem Vektorraum $\mathbf{K}\left(=\mathbf{R}^{\mathbf{4}}\right)$ wird durch die lineare Abbildung $I(x)=i x$ mit $I \circ I=-I d$ gegeben. Die unitäre Gruppe $U_{(2)}$ ist im folgenden immer als die Untergruppe der Elemente von $\mathbf{S O}(4)$, die mit $I$ vertauschbar sind, aufzufassen. Es folgt

$$
\mathbf{U}(2)=\alpha\left(\mathbf{S}_{1} \times \mathbf{S}_{3}\right) .
$$

Wir definieren den Homomorphismus $\mu$ von $\mathbf{U}(2)$ auf SO (3) als die Beschränkung von $\lambda^{(2)}$ auf $\mathbf{U}(2)$. Der Kern von $\mu$ ist gleich $\alpha\left(\mathbf{S}_{1} \times 1\right)$, ist also isomorph zu $\mathbf{S}_{1}$. Man hat eine exakte Sequenz

$$
0 \rightarrow \mathbf{S}_{1} \rightarrow \mathbf{U}_{(2) \stackrel{\mu}{\rightarrow}} \mathbf{S O}(3) \rightarrow 0 .
$$

Faßt man $\mathbf{U}(2)$ als Gruppe von $2 \times 2$-reihigen komplexen Matrizen auf, dann ist $\alpha\left(\mathbf{S}_{1} \times \mathbf{l}\right)$ die Untergruppe der skalaren Matrizen. $\mu$ induziert einen Isomorphismus von $\mathbf{P} \mathbf{U}_{(2)}$, der projektiv-unitären Gruppe, auf SO (3).

Der in 1.2 betrachtete maximale Torus $\alpha\left(\mathbf{S}_{1} \times \mathbf{S}_{1}\right)=\mathbf{S O}(2) \times \mathbf{S O}(2)$ von SO (4) ist in $\mathbf{U}(2)$ enthalten. Damit wird $\mu$ beschränkt auf diesen Torus wie $\lambda^{(2)}$ durch $u=t_{2}-t_{1}$ gegeben.

1.4. Mit $\mathbf{S}_{\mathbf{2}}$ bezeichnen wir die Sphäre der Einheitsvektoren im $\mathbf{R}^{3}$, der wie in 1.2 mit dem Raum der Quaternionen mit verschwindendem Realteil identifiziert wird. Die Gruppe $\mathbf{S}_{3}$ operiert nach (3) auf $\mathbf{S}_{2}$. Die Isotropiegruppe des Punktes $(1,0,0) \in \mathbf{S}_{2}$, d. h. des Punktes $i \in \mathbf{K}$, ist $\mathbf{S}_{1}$. Damit ist $\mathbf{S}_{3} / \mathbf{S}_{1} \cong \mathbf{S}_{\mathbf{2}}$, und wir haben die Projektion

$$
\hat{h}: \mathbf{S}_{\mathbf{3}} \rightarrow \mathbf{S}_{2}, \hat{h}(q)=q i q^{-1} .
$$

Die Gruppe $\mathbf{U}(2)$ operiert als Untergruppe von $\mathbf{S O}(4)$ auf $\mathbf{R}^{4}(=\mathbf{K})$ und damit auf $\mathbf{S}_{3} \subset \mathbf{K}$. Die Isotropiegruppe von $(1,0,0,0) \in \mathbf{S}_{3}$, d. h. von $\mathbf{I} \in \mathbf{K}$, ist $1 \times 1 \times S O(2)$. Damit ist $\mathbf{U}(2) / 1 \times 1 \times S O(2) \cong S_{3}$, und wir haben die Projektion

$$
p_{1}: \mathbf{U}(\mathbf{2}) \rightarrow \mathbf{S}_{3} .
$$


$\mathbf{U}(2)$ operiert vermöge (6) auf $S_{2}$. Die Isotropiegruppe von $(1,0,0) \in S_{2}$, d. h. von $i \in K$, ist SO (2) $\times \mathbf{S O}(2)$. Damit ist $\mathbf{U}(2) / \mathbf{S O}(2) \times \mathbf{S O}(2) \cong \mathbf{S}_{2}$, und wir haben die Projektion

$$
p_{2}: \mathbf{U}(2) \rightarrow \mathbf{S}_{2} .
$$

Vermöge (8) und $\left(8^{*}\right)$ hat man eine natürliche Projektion

$$
h: \mathbf{S}_{3} \rightarrow \mathbf{S}_{2}
$$

mit $h \circ p_{1}=p_{2}$. Das ist die sogenannte Hopfsche Abbildung. Es ist $h(q)$ $=q^{-1} \cdot i \cdot q$, wie man leicht kontrolliert. Also ist

$$
h(q)=\hat{h}\left(q^{-1}\right) .
$$

SO (4)/SO (2) $\times$ SO (2), der Raum der orientierten 2-dimensionalen Teilräume durch den Nullpunkt des $\mathbf{R}^{4}$, werde mit $\Sigma$ bezeichnet. $\Sigma$ ist bekanntlich homöomorph zu $\mathbf{S}_{2} \times \mathbf{S}_{2}$. Wir geben einen Homöomorphismus explizit an $((4),(7))$ :

$$
\begin{aligned}
\Sigma & =\alpha\left(\mathbf{S}_{3} \times \mathbf{S}_{3}\right) / \alpha\left(\mathbf{S}_{1} \times \mathbf{S}_{1}\right) \cong\left(\mathbf{S}_{3} \times \mathbf{S}_{3}\right) /\left(\mathbf{S}_{1} \times \mathbf{S}_{1}\right) \\
& \cong\left(\mathbf{S}_{3} / \mathbf{S}_{1}\right) \times\left(\mathbf{S}_{3} / \mathbf{S}_{1}\right) \cong \mathbf{S}_{2} \times \mathbf{S}_{2} .
\end{aligned}
$$

Damit haben wir zwei Projektionen $q_{1}, q_{2}$ von $\Sigma$ auf $\mathbf{S}_{2}$ definiert. Es sei $p$ die natürliche Projektion von SO(4) auf $\Sigma$. Die Abbildung $q_{r} \circ p(r=1,2)$ ist gleich $\lambda^{(r)}$ gefolgt von SO $(3) \rightarrow \mathbf{S}_{2}$, (vgl. $\left.\left(3^{*}\right),(7)\right)$. Vermöge $\lambda^{(r)}$ operiert SO (4) transitiv auf $\mathbf{S}_{2}$. Die Gruppe aller $g \in \mathbf{S O}(4)$, für die $\lambda^{(r)}(g)$ den Punkt $(1,0,0) \in \mathbf{S}_{2}$ festhält, ist $\alpha\left(\mathbf{S}_{1} \times \mathbf{S}_{3}\right)=\mathbf{U}(2)$ bzw, $\alpha\left(\mathbf{S}_{3} \times \mathbf{S}_{1}\right)$. Wir haben

$$
\text { SO (4) } / \mathbf{U}(2)=\alpha\left(\mathbf{S}_{3} \times \mathbf{S}_{3}\right) / \alpha\left(\mathbf{S}_{1} \times \mathbf{S}_{3}\right) \cong \mathbf{S}_{3} / \mathbf{S}_{1} \cong \mathbf{S}_{2} \text {. }
$$

Die Projektion $q_{1}$ kann wegen (12) auch definiert werden als die kanonische Abbildung von $\Sigma$ auf $\mathbf{S O}(4) / \mathbf{U}(2),(\mathbf{U}(2)>\mathbf{S O}(2) \times \mathbf{S O}(2))$. Entsprechend für $q_{\mathrm{2}}$.

Die Sphäre $\mathbf{S}_{3}$ ist kanonisch orientiert (als Rand der orientierten Vollkugel des dureh $d x_{1} \wedge d x_{2} \wedge d x_{3} \wedge d x_{4}$ orientierten $\left.\mathbf{R}^{4}\right) . \pi_{3}\left(\mathbf{S}_{3}\right)$ ist kanonisch isomorph mit $\mathbf{Z}$. Die Abbildung $h$ induziert einen Isomorphismus $\pi_{3}\left(\mathbf{S}_{3}\right) \rightarrow \pi_{3}\left(\mathbf{S}_{2}\right)$. Damit ist $\pi_{3}\left(\mathbf{S}_{2}\right)$ kanonisch isomorph mit $\mathbf{Z}$. Für die Definition des letzten kanonischen Isomorphismus haben wir $h$ genommen, nicht $\hat{h}$ [siehe (7)]. Der Homöomorphismus $\left(q_{1}, q_{2}\right)$ von $\Sigma$ auf $\mathbf{S}_{2} \times \mathbf{S}_{2}$ induziert jetzt einen kanonischen Isomorphismus

$$
\varrho: \pi_{3}(\Sigma) \rightarrow \mathbf{Z}+\mathbf{Z}
$$

Dabei ist zu beachten, daß die Abbildung (11) von $\mathbf{S}_{3} \times \mathbf{S}_{3}$ auf $\Sigma$ einen Isomorphismus $\varrho^{\prime}$ von $\mathbf{Z}+\mathbf{Z}$ auf $\pi_{3}(\Sigma)$ induziert. $\varrho \circ \varrho^{\prime}$ ist aber nicht die Identität, sondern die Abbildung $(a, b) \rightarrow(-a,-b)$, beachte $(10)$.

1.5. Satz. Es sei $\lambda^{(n)}: \mathbf{S O}(4) \rightarrow \mathbf{S O}(3)$ der in 1.2 definierte Homomorphismus. Die charakteristischen Klassen der $\lambda^{(r)}$-Erweiterung eines SO(4)-Prinzipalfaserbiindels $\xi$ sind

i)

ii)

iii)

$$
\begin{aligned}
p_{1}\left(\lambda_{*}^{(r)} \xi\right) & =p_{1}(\xi)-2(-1)^{r} W_{4}(\xi) \\
w_{2}\left(\lambda_{*}^{(r)} \xi\right) & =w_{2}(\xi) \\
W_{3}\left(\lambda_{*}^{(r)} \xi\right) & =W_{3}(\xi)
\end{aligned}
$$


Beweis: Wir setzen $\lambda^{(r)}$ gleich $\lambda$ und $(-1)^{r}$ gleich $\varepsilon$. Für den maximalen Torus SO (2) $\times$ SO (2) von SO (4) schreiben wir kurz $T$. Am Schluß von 1.2 wurde das Verhalten von $\lambda$ auf $T$ angegeben. Nach [2], Theorem 10.3, ist dann in reeller Cohomologie die folgende formale Rechnung legitimiert.

$$
\begin{aligned}
p_{1}\left(\lambda_{*} \xi\right)=u^{2}=\left(t_{2}-\varepsilon t_{1}\right)^{2} & =\left(t_{1}^{2}+t_{2}^{2}\right)-2 \varepsilon t_{1} t_{2} \\
& =p_{1}(\xi)-2 \varepsilon W_{4}(\xi) .
\end{aligned}
$$

Das ergibt i) in reeller Cohomologie.

$\Sigma=\mathbf{S O}(4) / T$ ist homöomorph zu $\mathbf{S}_{2} \times \mathbf{S}_{2}$, also $H^{1}\left(\mathbf{S O}(4) / T, \mathbf{Z}_{2}\right)=0$. Einfache Anwendung der Spektralsequenz ergibt, da $B$ in dem Faserbündel $\left(E_{\xi} / T, B_{\xi}, \mathbf{S O}(4) / T, \pi\right)$ der Homomorphismus $\pi^{*}$ von $H^{2}\left(B_{\xi}, \mathbf{Z}_{2}\right)$ in $H^{2}\left(E_{\xi} / T, \mathbf{Z}_{2}\right)$ injektiv ist. Deshalb ist in $\mathbf{Z}_{\mathbf{2}}$-Cohomologie die folgende Rechnung legitimiert (vgl. [2])

$$
w_{2}\left(\lambda_{*} \xi\right)=u=t_{2}-\varepsilon t_{1}=t_{1}+t_{2}=w_{2}(\xi) .
$$

Damit ist ii) bewiesen. iii) folgt, da für $\xi$ und $\lambda_{*} \xi$ gilt (1.1): $W_{3}=\delta_{*} w_{2}$. Für $\xi$ und $\lambda_{*} \xi$ ist bekanntlich [2] die $\mathbf{Z}_{2}$-Reduktion von $p_{1}$ gleich $u_{2}^{2}$. Aus

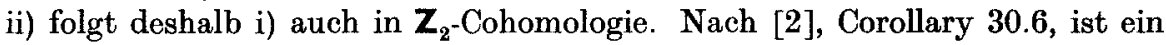
Element von $H^{*}\left(B_{\mathbf{S O}}(k), \mathbf{Z}\right)$ durch seine kanonischen Bilder in $H^{*}\left(B_{\mathbf{S O}}(k), \mathbf{R}\right)$ und $H^{*}\left(B_{\mathbf{S O}(k)}, \mathbf{Z}_{2}\right)$ bestimmt $\left.{ }^{2}\right)$. Wendet man dies auf die beiden Seiten von i) für den Fall an, daß $\xi$ das universelle Bündel ist, dann erhält man i) in ganzzahliger Cohomologie.

1.6. Satz. Es sei $\mu$ der in 1.3 definierte Homomorphismus von $\mathbf{U}(2)$ auf SO(3). Dann hat man für die charalteristischen Klassen der $\mu$-Erweiterung eines $\mathbf{U}(2)$-Prinzipalfaserbündels $\xi$

i)

ii)

iii) $p_{1}\left(\mu_{*} \xi\right)=c_{1}(\xi)^{2}-4 c_{2}(\xi)$,

$w_{2}\left(\mu_{*} \xi\right)$ ist die $\mathbf{Z}_{2}$-Reduktion von $c_{1}(\xi)$,

$W_{3}\left(\mu_{*} \xi\right)=0$.

Beweis: Nach [2], Theorem 10.3, ist die folgende formale Rechnung in ganzzahliger Cohomologie legitimiert, bei der man das Verhalten von $\mu$ auf dem maximalen Torus von $\mathbf{U}(2)$ zu berücksichtigen hat (vgl. den Schluß von 1.3).

$$
p_{1}\left(\mu_{*} \xi\right)=u^{2}=\left(t_{2}-t_{1}\right)^{2}=\left(t_{1}+t_{2}\right)^{2}-4 t_{1} t_{2} .
$$

Das ergibt i). In $\mathbf{Z}_{2}$-Cohomologie hat $\operatorname{man} w_{2}\left(\mu_{*} \xi\right)=u=t_{2}-t_{1}=t_{1}+t_{2}$ $=c_{1}(\xi)$. Das ergibt ii). Da $\delta_{*} w_{2}=W_{3}$ und $\delta_{*}$ auf $\mathbf{Z}_{2}$-Reduktionen ganzzahliger Klassen verschwindet, folgt iii).

\section{\$ 2. Das zweite Hindernis in $\mathbf{S}_{\mathbf{2}}$-Faserbündeln}

2.1. Für ein $\mathbf{U}(2)$-Prinzipalfaserbündel $\xi$ bezeichne $\xi^{\prime}$ das assoziierte Faserbündel mit $\mathbf{S}_{3}$ als Faser. Ferner sei $\xi^{\prime \prime}$ das zur $\mu$-Erweiterung von $\xi$ assoziierte Faserbündel mit $S_{2}$ als Faser (vgl. 1.3 und 1.4). Wie leicht zu sehen (1.1), ist

$$
E_{\xi^{\prime}}=E_{\xi} / 1 \times 1 \times \mathbf{S O}(2) \text { und } E_{\xi^{\prime \prime}}=E_{\xi} / \mathbf{S O}(2) \times \mathbf{S O}(2) \text {. }
$$

2) $H^{*}\left(B_{\mathbf{S O}}(k), \mathbf{Z}_{2}\right)$ hat nur Torsionselemente der Ordnung 2. 
Man hat die natürliche Projektion $h$ von $E_{\xi^{\prime}}$ auf $E_{\xi^{\prime \prime}}$ [siehe 1.4 (9)]. In der Tat ist $E_{\xi^{\prime}}$ ein Prinzipalfaserbündel über $E_{\xi^{\prime \prime}}$ mit Faser und Gruppe (SO (2) $\times$ $\times \mathbf{S O}(2)) / 1 \times 1 \times \mathbf{S O}(2) \cong \mathbf{S O}(2)$. Wegen der Darstellung (1) von $E_{\xi^{\prime}}$. hat man über $E_{\xi^{\prime \prime}}$ in kanonischer Weise ein geordnetes Paar von SO (2)-Bündeln, deren erstes mit $\gamma(\xi)$ bezeichnet werde. Wir fassen $\gamma(\xi)$ als $\mathbf{U}(1)$-Bündel auf $(\mathbf{S O}(2)$ $=\mathbf{U}(1)) . \gamma(\xi)$ ist in natürlicher Weise mit dem Faserbündel $\left(h: E_{\xi^{\prime}} \rightarrow E_{\xi^{\prime \prime}}\right)$ zu identifizieren. Die gemeinsame Basis von $\xi, \xi^{\prime}, \xi^{\prime \prime}$ werde mit $B$ bezeichnet.

Wir nehmen jetzt an, da $B$ ein endlicher Zellenkomplex ist. $B^{r}$ sei das $r$-dimensionale Gerüst von $B$. Es gibt Schnitte von $\xi^{\prime}$ über $B^{3}$, jeder derartige Schnitt definiert eine Hindernis-Cohomologieklasse, die als Element von $H^{4}(B, \mathbf{Z})$ aufzufassen ist $\left.^{3}\right)$, da die dritte Homotopiegruppe von $\mathbf{S}_{3}$ zu $\mathbf{Z}$ kanonisch isomorph ist (1.4). Die Hindernisse aller Schnitte von $\xi^{\prime}$ über $B^{3}$ sind gleich $c_{2}(\xi)$, dem ersten Hindernis von $\xi^{\prime}$ (vgl. 1.1).

Jeder Schnitt $s$ von $\xi^{\prime}$ induziert den Schnitt $h \circ s$ von $\xi^{\prime \prime}$. Also besitzt auch $\xi^{\prime \prime}$ Schnitte über $B^{3}$, in Ubereinstimmung mit 1.6 iii): Die StiefelWhitneysche Klasse $W_{3}$ eines $\mathbf{S O}(3)$-Bündels über $B$ ist nämlich gleich dem ersten Hindernis des assoziierten $\mathbf{S}_{2}$-Faserbündels. Jeder Schnitt $f$ von $\xi^{\prime \prime}$ über $B^{3}$ definiert ein (zweites) Hindernis $\Gamma(f)$, das als Element von $H^{4}(B, \mathbf{Z})$ aufgefaßt werden $\mathrm{kann}^{3}$ ), da die dritte Homotopiegruppe von $\mathbf{S}_{\mathbf{2}}$ zu $\mathbf{Z}$ kanonisch isomorph ist (1.4). Die Klasse $\Gamma(f)$ hängt im allgemeinen von $f \mathrm{ab}$. Wenn $s$ ein Schnitt von $\xi^{\prime}$ über $B^{3}$ ist, dann ist

$$
\Gamma(h \circ s)=c_{2}(\xi) \text {. }
$$

Der folgende Satz stammt von KunderT [11].

2.2. Satz. Ein Element $\Gamma$ von $H^{4}(B, Z)$ tritt dann und nur dann als Hindernis eines Schnittes von $\xi^{\prime \prime}$ über $B^{3}$ auf, wenn es ein $d \in H^{2}(B, \mathbf{Z})$ gibt, so daß

$$
\Gamma=d^{2}+d \cdot c_{1}(\xi)+c_{2}(\xi) .
$$

Beweis: Sei $d \in H^{2}(B, \mathbf{Z})$ und $\delta$ ein $\mathbf{U}(1)$-Prinzipalfaserbündel mit der ersten Chernschen Klasse $d$ (vgl. [6], Satz 4.3.1). Dann ist $\xi \otimes \delta$ wieder ein $\mathbf{U}(2)$-Bündel und

$$
c_{2}(\xi \otimes \delta)=d^{2}+d \cdot c_{1}(\xi)+c_{2}(\xi), \quad \text { vgl. [6], S. 67, Bemerk. }
$$

Aus $\xi^{\prime \prime}=(\xi \otimes \delta)^{\prime \prime}$ [siehe $1.3(6)$ ] folgt wegen (2), daß $c_{2}(\xi \otimes \delta)$ als Hindernis auftritt. Der Satz ist noch in umgekehrter Richtung zu beweisen: Wir verwenden die Bezeichnungen von 2.1, und $\delta$ sei wieder ein $\mathbf{U}$ (1)-Prinzipalfaserbündel über $B$. Ferner sei $\varrho$ die Projektion von $E_{\xi^{\prime \prime}}=E_{(\xi \otimes \delta)^{\prime \prime}}$ auf $B$. Utber $\boldsymbol{E}_{\xi^{\prime \prime}}$ hat man die $\mathbf{U}(1)$-Bündel $\gamma(\xi), \gamma(\xi \otimes \delta), \varrho^{*} \delta$, die in folgender Beziehung stehen

$$
\gamma(\xi \otimes \delta)=\gamma(\xi) \otimes \varrho^{*} \delta
$$

Nun sei $f$ ein Schnitt von $\xi^{\prime \prime}$ über $B^{3}$ und $s$ ein Schnitt von $\xi^{\prime}$ über $B^{3}$. Dann ist $c_{1}\left(f^{*} \gamma(\xi)\right)$ gleich der Differenz-Cohomologieklasse von $h \circ s$ und $f$, [vgl. [4], S. 114, Formel (14)]. Dieses Resultat wird auf $\xi \otimes \delta$ angewandt: Wenn $g$

8) Man beachte, daß die Strukturgruppe zusammenhängend ist. 
ein Schnitt von $(\xi \otimes \delta)^{\prime}$ und $\tilde{g}$ der induzierte Schnitt von $(\xi \otimes \delta)^{\prime \prime}$ ist, dann ist $c_{1}\left(f^{*} \gamma(\xi)\right)+c_{1}(\delta)$ wegen (4) die Differenz-Cohomologieklasse von $\tilde{g}$ und $f$. Wählt man nun für $\delta$ das $\mathbf{U}(1)$-Bündel über $B$ mit $c_{1}(\delta)=-c_{1}\left(f^{*} \gamma(\xi)\right)$, dann verschwindet die Differenz-Cohomologieklasse von $\tilde{g}$ und $f$. Dann ist $\Gamma(f)$ $=\Gamma(\tilde{g})$, und $\Gamma(f)$ ist also von der Gestalt (3), nämlich

$$
\Gamma(f)=d^{2}+d c_{1}(\xi)+c_{2}(\xi),
$$

wo $d=c_{1}(\delta)$ gleich der Differenz-Cohomologieklasse von $f$ und $h \circ s$ ist.

2.3. Wir haben in 1.3 die exakte Sequenz (6) betrachtet. Zu $\mu$ gehört eine Faserabbildung $\varrho(\mu): B_{\mathbf{U}(2)} \rightarrow B_{\mathbf{S O}(3)}$ der universellen Räume mit dem Eilenberg-MacLaneschen Raum $K(\mathbf{Z}, 2)=B_{\mathbf{S}_{1}}$, dem unendlich-dimensionalen komplexen projektiven Raum, als Faser. (Vgl. [1], § 22 oder [3], §1). Die zugehörige Spektralsequenz zeigt, daß das (einzige) Hindernis gegen einen Schnitt in diesem Faserraum die universelle Stiefel-Whitneysche Klasse $W_{3}$ ist. Daraus folgt auf übliche Weise, daß ein SO (3)-Prinzipalfaserbündel $\eta$ dann und nur dann die $\mu$-Erweiterung eines $\mathbf{U}(2)$.Prinzipalfaserbündels ist, wenn $W_{3}(\eta)=\mathbf{0}$ (vgl. 1.6 für ,nur dann").

2.4. Wir betrachten ein SO (3)-Prinzipalfaserbündel $\eta$ und das assoziierte Bündel $\eta^{\prime \prime}$ mit $\mathbf{S}_{2}$ als Faser. Die Basis $B$ von $\eta$ und $\eta^{\prime \prime}$ sei ein endlicher Zellenkomplex. Das erste Hindernis gegen einen Schnitt von $\eta^{\prime \prime}$ ist $W_{3}(\eta) \in H^{3}(B, Z)$. Wir nehmen an, daB $W_{3}(\eta)=0$. Dann gibt es Schnitte von $\eta^{\prime \prime}$ über $B^{3}$. Jedem derartigen Schnitt ist ein (zweites) Hindernis $\in H^{4}(B, Z)$ zugeordnet. $\left[\pi_{3}\left(\mathbf{S}_{2}\right)\right.$ ist kanonisch isomorph zu $\mathbf{Z}(1.4)$. $]$ Es stellt sich die Frage, welche Elemente von $H^{4}(B, \mathbf{Z})$ als Hindernisse von Schnitten von $\eta^{\prime \prime}$ über $B^{3}$ auftreten.

2.5. Satz. Es sei $\eta$ ein SO(3)-Prinzipalfaserbündel über einem endlichen Zellenkomplex B. Es sei $W_{3}(\eta)=0$. Das assaziierte Faserbündel $\eta^{\prime \prime}$ mit $\mathbf{S}_{\mathbf{2}}$ als Faser hat dann über $B^{3}$ einen Schnitt. Es treten genau die Elemente $\widetilde{\Gamma}$ $\in H^{4}(B, Z)$ als Hindernis multipliziert mit 4 eines solchen Schnittes auf, welche folgendermaßen dargestellt werden können

$$
\tilde{\Gamma}=x^{2}-p_{1}(\eta), x \in H^{2}(B, \mathbf{Z}), x \equiv w_{2}(\eta) \bmod 2 .
$$

Insbesondere ist also jedes Element $\widetilde{\Gamma}$ der Form $(5)$ in $H^{\mathbf{4}}(B, \mathbf{Z})$ durch 4 teilbar.

Korollar. Wenn $H^{4}(B, Z)$ keine 2-Torsion hat, so hat $\eta^{\prime \prime}$ genau dann einen Schnitt über $B^{4}$, wenn es ein $x \in H^{2}(B, \mathbf{Z})$ gibt, für das $x^{2}=p_{1}(\eta)$ und dessen $\mathbf{Z}_{2}$ Redultion gleich $w_{\mathbf{2}}(\eta)$ ist.

Beweis des Satzes: Wir wählen nach 2.3 ein $\mathbf{U}(2)$-Prinzipalfaserbündel $\xi$ mit $\mu_{*} \xi=\eta$. Dann ist $\xi^{\prime \prime}=\eta^{\prime \prime}$, und die gesuchten möglichen Hindernisse sind durch 2.2 (3) gegeben. Nun ist

$$
4\left(d^{2}+d c_{1}(\xi)+c_{2}(\xi)\right)=\left(2 d+c_{1}(\xi)\right)^{2}-\left(c_{1}(\xi)^{2}-4 c_{2}(\xi)\right)
$$

Der Satz folgt jetzt aus 1.6.

Bemerkung: 2.5 bestätigt die Vermutung von H. HoPF, daß die Koeffizienten, die in seinem quadratischen Polynom für die möglichen zweiten Hindernisse auftraten, mit charakteristischen Klassen zusammenhängen 
(vgl. [9], S. 121 unten). Das besagte quadratische Polynom findet sich in der vorliegenden Arbeit in $2.2(3)$ und $\left(3^{*}\right)$ wieder. Die Koeffizienten $c_{1}(\xi), c_{2}(\xi)$ hängen von der Wahl des $\mathbf{U}(2)$-Bündels $\xi$ mit $\mu_{*} \xi=\eta$ ab. $c_{1}(\xi) \bmod .2$ und $c_{1}(\xi)^{2}-4 c_{2}(\xi)$ sind aber wegen 1.6 unabhängig von der Wahl von $\xi$. Man kann direkt beweisen, daß $c_{1}(\xi)$ in 2.2 (3) gleich der von HopF betrachteten Klasse $\omega(h \circ s, h \circ s)$ ist. Bezeichnet man noch die Differenz-Cohomologieklasse von $f$ und $h \circ s$ mit $\alpha(f, h \circ s)$, dann geht $2.2\left(3^{*}\right)$ über in

$$
\Gamma(f)=\alpha(f, h \circ s)^{2}+\alpha(f, h \circ s) \cdot \omega(h \circ s, h \circ s)+\Gamma(h \circ s) .
$$

Das ist genau die Formel (7.4) von [9], die von HopF allerdings unter allgemeineren Voraussetzungen über die Strukturgruppe bewiesen wurde (vgl. auch [10]). Inzwischen ist eine Arbeit von WU WEN-Tsun über die erwähnte Vermutung erschienen, die uns unzugänglich ist. Ferner haben wir kürzlich (American Mathematical Society, Notices, February 1958, S. 27) eine Note von W. S. MASSEY gesehen, in der das obige Korollar ausgesprochen wird.

\section{3. Das zweite Hindernis in gewissen $\mathbf{S}_{2} \times \mathbf{S}_{2}$-Faserbündeln}

3.1. Es sei $\xi$ ein SO (4)-Prinzipalfaserbündel. Die Basis $B$ von $\xi$ sei ein endlicher Zellenkomplex. Das assoziierte Faserbündel $\bar{\xi}$ mit der Stiefelschen Mannigfaltigkeit SO (4)/1 $\times 1 \times \mathbf{S O}(2)$ der 2-Beine als Faser hat einen Schnitt über dem 2-dimensionalen Gerüst $B^{2}$, und das Hindernis gegen Fortsetzung eines solchen Schnittes auf $B^{3}$ ist gleich $W_{3}(\xi) \in H^{3}(B, \mathbf{Z})$. Ferner betrachten wir das zu $\xi$ assoziierte Faserbündel $\bar{\xi}$ mit $\Sigma=\mathbf{S O}(4) / \mathbf{S O}(2) \times \mathbf{S O}(2)$ als Faser und schließlich die beiden Faserbündel $\xi_{1}, \xi_{2}$ mit $S_{2}$ als Faser, die zur $\lambda^{(1)}$. bzw. $\lambda^{(2)}$-Erweiterung von $\xi$ assoziiert sind. Wir haben $(1.1,1.4)$

$$
\begin{array}{ll}
E_{\xi}=E_{\xi} / \mathbf{1} \times 1 \times \mathbf{S O}(2), & E_{\xi}=E_{\xi} / \mathbf{S O}(2) \times \mathbf{S O}(2) \\
E_{\xi_{1}}=E_{\xi} / \mathbf{U}(2), & E_{\xi_{\xi}}=E_{\xi} / \alpha\left(\mathbf{S}_{3} \times \mathbf{S}_{1}\right)
\end{array}
$$

und natürliche Projektionen (1.4)

$$
\begin{aligned}
& \varphi: E_{\bar{\xi}} \rightarrow E_{\bar{\xi}} \\
& q_{1}: E_{\bar{\xi}} \rightarrow E_{\xi_{1}}, \quad q_{2}: E_{\bar{\xi}} \rightarrow E_{\xi_{2}} .
\end{aligned}
$$

Jeder Schnitt 8 von $\bar{\xi}$ (über einer Teilmenge von $B$ ) induziert Schnitte $q_{1} \circ s$, $q_{2} \circ s$ von $\xi_{1}$ bzw. $\xi_{2}$. Umgekehrt gibt es zu jedem Paar $f_{1}, f_{2}$, wo $f_{r}$ ein Schnitt von $\xi_{r}$ ist, genau einen Schnitt $s$ von $\overline{\bar{\xi}} \operatorname{mit}\left(f_{1}, f_{2}\right)=\left(q_{1} \circ s, q_{2} \circ s\right)$. Ferner stellen wir fest, daß jeder Schnitt $s$ von $\bar{\xi}$ den Schnitt $\varphi \circ s$ von $\bar{\xi}$ induziert. Das Hindernis gegen einen Schnitt über $B^{3}$ ist für jedes der Bündel $\bar{\xi}, \xi_{1}, \xi_{2}$ gleich $W_{3}(\xi)$ [siehe 1.5 iii)]. Wir setzen jetzt voraus, da $\beta W_{3}(\xi)=0$. Dann hat jedes der Bündel $\bar{\xi}, \bar{\xi}, \xi_{1}, \xi_{2}$ einen Schnitt über $B^{3}$, und es tritt die Frage auf, welche 4-dimensionalen Cohomologieklassen als Hindernisse von Schnitten über $B^{3}$ (gegen die Fortsetzung auf $B^{4}$ ) auftreten. Diese Frage ist durch 2.5 für die $S_{2}$-Faserbündel $\xi_{1}, \xi_{2}$ gelöst, und damit läßt sie sich für $\bar{\xi}$ beantworten :

3.2. Das Hindernis eines Schnittes $s$ von $\xi$ über $B^{3}$ ist eine 4-dimensionale Cohomologieklasse mit Koeffizienten in der Homotopiegruppe $\pi_{3}(\Sigma)$, welche 
kanonisch isomorph zu $\mathbf{Z}+\mathbf{Z}$ ist [1.4 (13)]. Also läßt sich die HindernisCohomologieklasse des Schnittes $s$ in bestimmter Weise als Element von $H^{4}(B, \mathbf{Z}+\mathbf{Z})=H^{4}(B, \mathbf{Z})+H^{4}(B, \mathbf{Z})$ auffassen und damit als Paar von Elementen aus $H^{4}(B, \mathbf{Z})$, dessen $r$-te Komponente $(r=1,2)$ offenbar gleich dem Hindernis von $q_{r} \circ s$ in $\xi_{r}$ ist. Hier hat $q_{r}$ die in (2) angegebene Bedeutung. Es folgt, daB ein Paar $(a, b) \in H^{4}(B, \mathbf{Z})+H^{4}(B, \mathbf{Z})$ dann und nur dann als Hindernis eines Schnittes von $\overline{\bar{\xi}}$ über $B^{3}$ auftritt, wenn $a$ bzw. $b$ Hindernis eines Schnittes von $\xi_{1}$ bzw. $\xi_{2}$ über $B^{3}$ ist. Anwendung von 2.5 auf $\xi_{1}, \xi_{2}$ ergibt unter Benutzung von 1.5 den folgenden Satz.

3.3. Satz. Es sei $\xi$ ein SO(4)-Prinzipalfaserbündel über einem endlichen Zellenkomplex B. Es sei $W_{3}(\xi)=0$. Das assoziierte Faserbündel $\vec{\xi}$ mit $\Sigma$, dem Raum der orientierten 2-dimensionalen Teilräume durch den Nullpunkt des $\mathbf{R}^{\mathbf{4}}$, als Faser hat dann über $B^{3}$ einen Schnitt, und es treten genau die Elemente $(\tilde{a}, \tilde{b}) \in H^{4}(B, \mathbf{Z})+H^{4}(B, \mathbf{Z})$ als Hindernis (vgl. 3.2) multipliziert mit 4 eines solchen Schnittes auf, welche folgendermaßen dargestellt werden können

$$
\begin{gathered}
(\tilde{a}, \tilde{b})=\left(x^{2}-p_{1}(\xi)-2 \cdot W_{4}(\xi), y^{2}-p_{1}(\xi)+2 \cdot W_{4}(\xi)\right), \\
\text { wobei } x, y \in H^{2}(B, \mathbf{Z}), \quad x \equiv y \equiv w_{2}(\xi) \bmod 2 .
\end{gathered}
$$

3.4. Wir betrachten nun das Faserbündel $\bar{\xi}$. Die natürliche Projektion von SO (4) $/ 1 \times 1 \times \mathbf{S O}_{(2)}$ auf $\Sigma$ induziert einen Isomorphismus der dritten Homotopiegruppen, wie sofort aus der exakten Homotopiesequenz folgt. Damit ist nach 1.4 (13) ein kanonischer Isomorphismus

$$
k_{1}: \pi_{3}(\mathbf{S O}(4) / 1 \times 1 \times \mathbf{S O}(2)) \rightarrow \mathbf{Z}+\mathbf{Z}
$$

gegeben, und das Hindernis eines Schnittes von $\bar{\xi}$ über $B^{3}$ kann wieder als Paar von Elementen aus $H^{4}(B, Z)$ aufgefaßt werden. Offenbar hat der Schnitt $s$ von $\bar{\xi}$ über $B^{3}$ dann das gleiche Hindernis wie der Schnitt $\varphi \circ s$ [vgl. 3.1 (2)] von $\bar{\xi}$ über $B^{3}$.

3.5. Satz. Es sei $\xi$ ein SO(4)-Prinzipalfaserbündel über einem endlichen Zellenkomplex $B$. Es sei $W_{3}(\xi)=0$. Das assoziierte Faserbïndel $\bar{\xi}$ mit der Stiefelschen Mannigfaltigkeit SO (4)/1 $\times 1 \times \mathbf{S O}(2)$ als Faser hat dann über $B^{3}$ einen Schnitt, und es treten genau die Elemente $(\tilde{a}, \tilde{b}) \in H^{4}(B, \mathbf{Z})+H^{4}(B, \mathbf{Z})$ als Hindernis multipliziert mit 4 eines solchen Schnittes auf, welche folgendermaßen dargestellt werden können

$$
\begin{gathered}
(\tilde{a}, \tilde{b})=\left(x^{2}-p_{1}(\xi)-2 W_{4}(\xi), x^{2}-p_{1}(\xi)+2 W_{4}(\xi)\right), \\
\text { wobei } x \in H^{2}(B, Z) \text { und } x \equiv w_{2}(\xi) \bmod 2 .
\end{gathered}
$$

Beweis: Die Stiefelsche Mannigfaltigkeit SO (4)/1 $\times 1 \times \mathrm{SO}_{(2)}$ ist homöomorph zu $\mathbf{S}_{3} \times \mathbf{S}_{2}$. Wir haben die natürliche Projektion

$$
q: \mathrm{SO}(4) / 1 \times 1 \times \mathrm{SO}(2) \rightarrow \mathrm{SO}(4) / 1 \times \mathrm{SO}(3) \cong \mathrm{S}_{3} .
$$

Ferner haben wir eine Projektion $q_{1}^{\prime}$ der Stiefelschen Mannigfaltigkeit auf $\mathbf{S}_{\mathbf{q}}$, die durch (vgl. 1.4)

$$
\mathrm{SO}(4) / 1 \times 1 \times \mathrm{SO}(2) \rightarrow \mathrm{SO}(4) / \mathrm{SO}(2) \times \mathrm{SO}(2) \stackrel{q_{1}}{\rightarrow} \mathrm{S}_{2}
$$


definiert wird. Wie leicht aus $1.2-1.4$ folgt, ist $\left(q_{1}, q\right)$ ein Homöomorphismus der Stiefelschen Mannigfaltigkeit auf $\mathbf{S}_{2} \times \mathbf{S}_{3}$, der einen Isomorphismus $k_{2}$ ihrer dritten Homotopiegruppe auf $\mathbf{Z}+\mathbf{Z}$ induziert (1.4).

$\xi^{\prime}$ sei das zu $\xi$ assoziierte Faserbündel mit $\mathbf{S}_{3}$ als Faser. Jeder Schnitt von $\xi^{\prime}$ über $B^{3}$ hat das Hindernis $W_{4}(\xi)$. Andererseits entsprechen die Schnitte von $\bar{\xi}$ über $B^{3}$ offenbar den Paaren $\left(f_{1}, f_{2}\right)$, wo $f_{1}$ ein Schnitt von $\xi_{1}$ über $B^{3}$ und $f_{2}$ ein Schnitt von $\xi^{\prime}$ über $B^{3}$ ist. Das 4-fache Hindernis von $f_{1}$ ist von der Gestalt $x^{2}-p_{1}(\xi)-2 W_{4}(\xi),\left(x \equiv w_{2}(\xi) \bmod 2\right)$, während das von $f_{2}$ gleich $4 W_{4}(\xi)$ ist. Wir haben den Automorphismus $k=k_{2} \circ k_{1}^{-1}$ von $Z+Z$ auf sich und behaupten $k(a, b)=(a, b-a)$. Dies folgt bei genauer Beachtung der am Schluß von 1.4 getroffenen Konventionen. Damit ist der Satz bewiesen.

\section{\$ 4. Das Tangentialbündel einer 4-dimensionalen Mannigfaltigkeit}

4.1. Es sei $M$ eine 4-dimensionale kompakte zusammenhängende orientierte differenzierbare Mannigfaltigkeit (versehen mit einer Riemannschen Metrik) und $\xi$ ihr tangentielles SO (4)-Prinzipalfaserbündel. Die charakteristischen Klassen von $\xi$ (vgl. 1.1) nennt man auch charakteristische Klassen von $M$. Sie werden mit $w_{2}(M), W_{3}(M), W_{4}(M), p_{1}(M)$ bezeichnet. Wir werden in diesem Abschnitt sehen, $\mathrm{da} B$ sie durch klassische Cohomologieinvarianten von $M$ bestimmt sind. Gleichzeitig erinnern wir noch einmal an einige bereits in der Einleitung erwähnte Begriffe.

Ein Element $y \in H^{4}(M, Z)$ ist durch seinen Wert $y[M]$ auf dem Grundzyklus der orientierten $M$ bestimmt. Wir haben zunächst

i)

$W_{4}(M)[M]$ ist die Eulersche Charaleteristik $e(M)$.

Es sei $T^{2}$ die Torsionsgruppe von $H^{2}(M, \mathbf{Z})$ und $H=H^{2}(M, \mathbf{Z}) / T^{2}$. Die Gruppe $H$ ist ein $b_{2}$-dimensionales Gitter, wobei $b_{i}$ die $i$-te Bettisehe Zahl von $M$ ist. $\mathrm{Da}(x y)[M]$ für $x, y \in H^{2}(M, \mathbf{Z})$ offenbar nur von den durch $x, y$ gegebenen Elementen von $H$ abhängt, erhalten wir eine symmetrische Bilinearform $S$ über $H$ von der Determinante \pm 1 (vgl. Einleitung). Als Form über dem reellen Vektorraum $H \otimes \mathbf{R}$ kann diese auf Diagonalform gebracht werden. Es sei $p^{+}$(bzw. $p^{-}$) die Anzahl der positiven (bzw, negativen) Diagonalkoeffizienten. Es ist dann $p^{+}+p^{-}=b_{2}$, während $p^{+}-p^{-}$der Index $\tau(M)$ ist. Es gilt

ii)

$$
p_{1}(M)[M]=3 \cdot \tau(M) .
$$

Diese Formel folgt aus der Cobordisme-Theorie von Thom [14]; (vgl. auch [6]).

Für einen Raum $X$ hat STEenrod für jedes $i(i \geqq 0)$ einen Homomorphismus $S q^{i}: H^{k}\left(X, \mathbf{Z}_{2}\right) \rightarrow H^{k+i}\left(X, \mathbf{Z}_{2}\right)$ definiert (Steenrodsche reduzierte Quadrate). Wenn $x \in H^{k}\left(X, \mathbf{Z}_{2}\right)$ und $i>k$, dann $\$ q^{i} x=0$. Für $x \in H^{i}\left(X, \mathbf{Z}_{2}\right)$ ist $S q^{i} x$ $=x^{2}$. Wenn $X$ eine zusammenhängende kompakte $n$-dimensionale Mannigfaltigkeit ist (nicht notwendigerweise orientierbar), dann wird für $i \leqq n$ der Homomorphismus $S q^{d}: H^{n-i}\left(X, \mathbf{Z}_{2}\right) \rightarrow H^{n}\left(X, \mathbf{Z}_{2}\right) \cong \mathbf{Z}_{2}$ wegen des Poincaréschen Dualitätssatzes durch Multiplikation mit einer Klasse $U_{i} \in H^{i}\left(X, Z_{2}\right)$ gegeben, $\mathrm{d} . \mathrm{h}$. es ist $S q^{i} x=U_{i} x$ für alle $x \in H^{n-i}\left(X, \mathbf{Z}_{2}\right)$. Die $U_{i}$ wurden 
von $W_{U}$ Wen-Tsun [16] eingeführt, der auch gezeigt hat, daß die $U_{i}$ Polynome in den Stiefel-Whitneyschen Klassen $w_{i}$ von $X$ sind (vgl. hierzu $[5,13$, 17]). In [5] wurden diese Polynome durch Reduktion mod 2 aus den Toddschen Polynomen erhalten. Wenn $X$ orientierbar ist, dann ist $w_{1}=0$; in diesem Fall verschwinden die $U_{i}$ für ungerades $i$, und es ist $U_{2}=w_{2}, U_{4}=w_{4}+w_{2}^{2}$, $U_{\mathbf{8}}=w_{4} w_{2}+w_{3}^{2}$.

Wir betrachten nun wieder unsere 4 -dimensionale $M$. Der Homomorphismus $x \rightarrow x^{2}$ von $H^{2}\left(M, \mathbf{Z}_{2}\right)$ in $H^{4}\left(M, \mathbf{Z}_{2}\right)$ ist gleich $S q^{2}$. Wie oben erwähnt, ist $U_{2}=w_{2}$, also gilt

$$
x^{2}=w_{2}(M) \cdot x \quad \text { für alle } x \in H^{2}\left(M, \mathbf{Z}_{2}\right) .
$$

$\mathrm{Zu}$ der exakten Koeffizientensequenz

$$
0 \rightarrow \mathbf{Z} \stackrel{2}{\rightarrow} \mathbf{Z} \rightarrow \mathbf{Z}_{\mathbf{2}} \rightarrow \mathbf{0}
$$

gehört die exakte Cohomologiesequenz

$$
H^{2}(M, \mathbf{Z}) \stackrel{2}{\rightarrow} H^{2}(M, \mathbf{Z}) \stackrel{r}{\longrightarrow} H^{2}\left(M, \mathbf{Z}_{2}\right) \stackrel{\delta_{*}}{\rightarrow} H^{3}(M, \mathbf{Z})
$$

Der Homomorphismus $r$ ist die $\mathbf{Z}_{2}$-Reduktion. Es ist

$$
r H^{2}(M, \mathbf{Z}) \cong H^{2}(M, \mathbf{Z}) / 2 H^{2}(M, \mathbf{Z}) \text {. }
$$

Wir betrachten die Inklusionen

$$
H^{2}\left(M, \mathbf{Z}_{2}\right) \supset r H^{2}(M, \mathbf{Z}) \supset r T^{2} .
$$

Aus dem Poincaréschen Dualitätssatz folgt leicht, daß $r H^{2}(M, Z)$ und $r T^{2}$ bezüglich des Cup-Produktes in $H^{2}\left(M, \mathbf{Z}_{2}\right)$ gegenseitige Annulatoren sind. Also: Für $z \in H^{2}\left(M, \mathbf{Z}_{2}\right)$ ist dann und nur dann $z x=0$ für alle $x \in r T^{2}$, wenn $z \in r H^{2}(M, \mathbf{Z})$.

$$
w_{2}(M) \text { ist die } \mathbf{Z}_{2^{-}} \text {Reduktion einer ganzzahligen Klasse. }
$$

Beweis: Wenn $x \in r T^{2}$, dann ist $x \boldsymbol{x}=0$. Wegen iii) ist auch $x \cdot w_{2}(M)=0$. Aus der obigen Annulierungseigenschaft folgt, daß $w_{2}(M) \in r H^{2}(M, Z)$.

Wegen eines oben erwähnten Isomorphismus ist $w_{2}(M)$ in natürlicher Weise als Element von $H^{2}(M, \mathbf{Z}) / 2 H^{2}(M, \mathbf{Z})$ aufzufassen. Bei dem natürlichen Homomorphismus von $H^{2}(M, \mathbf{Z}) / 2 H^{2}(M, Z)$ in $H / 2 H$ geht $w_{2}(M)$ in ein Element $W$ von $H / 2 H$ über. Diese Restklasse $W$ stimmt wegen iii) mit der in der Einleitung betrachteten Restklasse $W$ überein, d. h.

v)

$$
\$(w, x) \equiv S(x, x) \bmod 2 \text { für } x \in H \text { und } w \in W \subset H .
$$

Wie aus der Anschreibung der vorstehenden Formel ersichtlich ist, haben wir das Element $W$ von $H / 2 H$ als Restklasse, d. h. als Teilmenge von $H$ aufgefaßt. Wir führen wie in der Einleitung die Menge $\Omega$ aller ganzen Zahlen der Form $S(y, y),(y \in W)$, ein. Offensichtlich ist $\Omega$ auch gleich der Menge aller Zahlen $x^{2}[M]$ mit $x \subseteq H^{2}(M, Z)$ und $x \equiv w_{2}(M)$ mod 2. Weiter bemerken wir, da $\Omega$ dann und nur dann aus einem einzigen Element besteht, wenn $b_{2}(M)=0$. In diesem Fall ist $\Omega=\{0\}$.

Die Stiefel-Whitneysche Klasse $W_{3}(M) \in H^{3}(M, Z)$ ist gleich $\delta_{*} w_{2}(M)$ (vgl. 1.1). Aus iv) und der Exaktheit der obigen Cohomologiesequenz erhält man den folgenden Satz von WHTNeY [15]:

vi) Für eine kompakte orientierte 4-dimensionale $M$ verschwindet $W_{3}(M)$. 
Bemerkung: iv) und vi) lassen sich sofort auf kompakte orientierte $4 \mathrm{k}$ dimensionale $\boldsymbol{M}$ verallgemeinern. Es gilt, daß $U_{2 k}$ die $\mathbf{Z}_{2}$-Reduktion einer ganzzahligen Klasse ist und daß also $\delta_{*} U_{2 k}$ verschwindet. In einer 8-dimensionalen $M$ ist $U_{4}=w_{4}+w_{2}^{2}$. Da $u_{2}^{2}$ die $\mathbf{Z}_{2}$-Reduktion von $p_{1}$ ist (vgl. [2]), folgt für den Fall einer 8-dimensionalen $M$, daß $\delta_{*} w_{4}=W_{5}=0$.

4.2. Wir betrachten jetzt die zu dem Tangentialbündel $\xi$ assoziierten Faserbündel $\bar{\xi}$ und $\bar{\xi}$ (siehe 3.1). $\bar{\xi}$ ist das Bündel der (orthonormierten) 2-Beine und $\bar{\xi}$ das Bündel der orientierten Flächenelemente von $M$. Da $W_{3}(M)$ verschwindet, gibt es 2-Bein-Felder und auch Felder von Flächenelementen mit endlich vielen Singularitäten. Jede Singularität hat als „Index“ ein Paar ganzer Zahlen. Jedes Feld hat eine Indexsumme, die wieder ein Paar ganzer Zahlen ist. Welche Paare von ganzen Zahlen treten als Indexsumme eines Feldes von orientierten Flächenelementen mit endlich vielen Singularitäten auf? Dieselbe Frage stellt sich für Felder von 2-Beinen. Die Antworten sind in 3.3 und 3.5 enthalten. Sie lassen sich wegen 4.1 mit alleiniger Verwendung der Eulerschen Charakteristik $e=e(M)$ und der Poincaréschen Bilinearform $S$ formulieren. Durch $S$ sind die Zahlenmenge $\Omega$ und der Index $\tau=\tau(M)$ bestimmt.

4.3. Satz. Es sei $M$ eine 4-dimensionale kompakte orientierte differenzierbare Mannigfaltigkeit. Es gibt auf $M$ Felder von orientierten Flächenelementen mit endlich vielen Singularitäten. Ein Zahlenpaar $(a, b)$ tritt dann und nur dann als Indexsumme eines derartigen Feldes auf, wenn

$$
a=\frac{1}{4}(\alpha-3 \tau-2 e), \quad b=\frac{1}{4}(\beta-3 \tau+2 e) \text { mit } \alpha, \beta \in \Omega .
$$

Es gibt auf $M$ auch Felder von 2-Beinen mit endlich vielen Singularitäten. Ein Paar $(a, b)$ tritt dann und nur dann als Indexsumme eines solchen Feldes auf, wenn

$$
a=\frac{1}{4}(\alpha-3 \tau-2 e), \quad b=\frac{1}{4}(\alpha-3 \tau+2 e) \text { mit } \alpha \in \Omega .
$$

Die rechten Seiten von (1) und (2) sind immer ganzzahlig.

4.4. Korollar. Die Indexsumme $(a, b)$ aus (1) ist dann und nur dann unabhängig von der Wahl des Feldes, wenn die zweite Bettische Zahl von M verschwindet. Dann ist $\tau=0, \Omega=\{0\}$, und die invariante Indexsumme ist (-e/2, $+e / 2)$. Dasselbe gilt für (2).

4.5. Korollar. Es gibt auf ganz $M$ ein Feld orientierter Flächenelemente dann und nur dann, wenn $3 \tau+2 e$ und $3 \tau-2 e$ zu $\Omega$ gehören. Es gibt auf ganz $M$ ein 2-Bein-Feld dann und nur dann, wenn $e=0$ und $3 \tau \in \Omega$.

4.6. Die ganzen Zahlen der Form $\frac{1}{4}(\alpha-3 \tau-2 e), \alpha \in \Omega$, sind die mög. lichen Indexsummen (Hindernisse) für das Bündel $\xi_{1}$, das $E_{\xi} / \mathbf{U}_{(2)}$ als Totalraum hat (vgl. 1.4 und 3.1). Also läßt $M$ dann und nur dann eine fastkomplexe Struktur zu, wenn $\xi_{1}$ über ganz $M$ einen Schnitt hat (1.1). Das ist aber genau dann der Fall, wenn unter den möglichen Indexsummen die Zahl 0 vorkommt. Damit erhalten wir einen Satz von Wu Wev-Tsun ([18], S. 74) in folgender Fassung. 
Satz. Eine 4-dimensionale orientierte kompakte differenzierbare Mannigfaltigkeit läßt dann und nur dann eine fast-komplexe Struktur $z u$, wenn $3 \tau+2 e$ $\in \Omega$.

Bemerkung: Dieser Satz gilt für die orientierte Mannigfaltigkeit $\boldsymbol{M}$. Wir haben immer von dem SO (4)-Tangentialbündel Gebrauch gemacht. Nimmt man die entgegengesetzte Orientierung, dann bleibt $e$ fest, aber $\tau$ ändert sein Vorzeichen. $\Omega$ geht über in $-\Omega=\{\alpha:-\alpha \in \Omega\}$. Aus 4.5 und dem vorstehenden Satz folgt, daß $M$ dann und nur dann bezüglich beider Orientierungen eine fast-komplexe Struktur zuläBt, wenn sie ein Feld von orientierten Flächenelementen ohne Singularitäten besitzt. Für die komplexe projektive Ebene mit der üblichen Orientierung ist $3 \tau+2 e=3+6=9$ und $\Omega$ ist die Menge der ungeraden Quadratzahlen. Für die entgegengesetzte Orientierung hat man $3 \tau+2 e=3$. Nur für die erste Orientierung läßt die komplexe projektive Ebene eine fast-komplexe Struktur zu [7].

4.7. Nach 1.3 ist $\mu$ als die Beschränkung von $\lambda^{(2)}$ auf $\mathbf{U}_{(2)}$ definiert. Daraus folgt: Wenn $M$ mit einer fast-komplexen Struktur versehen ist, dann ist $\xi_{2}$ das $\mathbf{S}_{2}$-Faserbündel, das zur $\mu$-Erweiterung des tangentiellen $\mathbf{U}(2)$ Bündels von $M$ assoziiert ist (vgl. 3.1, $\xi$ ist immer das tangentielle SO (4)Bündel von $M$ ). Also ist $\xi_{2}$ das Bündel der komplexen Linienelemente der fastkomplexen $M$, und eine ganze Zahl $b$ tritt dann und nur dann als Indexsumme eines Feldes komplexer Linienelemente mit endlich vielen Singularitäten auf, wenn $b=\frac{1}{4}(\beta-3 \tau+2 e)$ mit $\beta \in \Omega$. Für die komplexe projektive Ebene treten die ganzen Zahlen der Form $\frac{1}{4}\left((2 k+1)^{2}-3+6\right)=k^{2}+k+1$ ( $k$ ganz) auf $[7,8]$. - Wie wir gesehen haben, ist ,,das Bündel $\xi_{2}$ der komplexen Linienelemente" mit PU (2) (= SO (3)) als Strukturgruppe unabhängig von der fast-komplexen Struktur und kann sogar definiert werden, wenn $M$ gar keine fast-komplexe Struktur besitzt. $\xi_{2}$ besitzt dann und nur dann einen Schnitt mit der Indexsumme $e$, wenn $M$ eine fast-komplexe Struktur zuläßt.

4.8. Für die von uns betrachteten $M$ ist die Existenz eines 1-Bein-Feldes über ganz $M$ mit $e=0$ gleichbedeutend, die Existenz eines 2-Bein-Feldes mit $e=0$ und $3 \tau \in \Omega$.

Satz. Die Existenz eines 3-Bein-Feldes, d.h. die Parallelisierbarkeit, ist äquivalent mit $e=\tau=0$ und $x^{2}=0$ für alle $x \in H^{2}\left(M, Z_{2}\right)$.

Beweis: Wenn $M$ parallelisierbar, dann verschwinden alle charakteristischen Klassen, also $e=\tau=0$ und $x^{2}=0\left(x \in H^{2}\left(M, Z_{2}\right)\right)$ wegen 4.1 i), ii), iii). Wenn umgekehrt $x^{2}=0$ für alle $x \in H^{2}\left(M, Z_{2}\right)$, dann ist $w_{2}(M)=0$ und das Tangentialbündel $\xi$ läBt Spin (4) $=\mathbf{S}_{3} \times S_{3}$ als Strukturgruppe zu ([2], $\left.\S 26\right)$. Das Hindernis gegen einen Schnitt in diesem Spin (4)-Bündel verschwindet, wenn $p_{1}(M)$ und $W_{4}(M)$ verschwinden, also wenn $e=\tau=0$. Wenn das Spin (4). Bündel einen Schnitt hat, dann auch $\xi$.

Eine $M$, die ein 1-Bein-Feld, aber kein 2-Bein-Feld zuläßt, ist uns nicht bekannt. 


\section{Literatur}

[1] Bonmt, A.: Sur la cohomologie des espaces fibrés principaux et des espaces homogènes de groupes de Lie compacts. Ann. of Math. 57, $115-207$ (1953), - [2] BorEL, A., and F. HinzenRdCH: Characteristic classes and homogeneous spaces. Part I: Amer. J. Math. 80, 458-538 (1958). Part II : Amer. J. Math. (erseheint demnächst) - [3] BoreL, A., et J. P. Skrre: Groupes de lie et puissances réduites de Steenrod. Amer. J. Math. 75, 409-448 (1953). - [4] HrrzeBruch, F.: Utbertragung einiger Sätze aus der Theorie der algebraischen Flächen auf komplexe Mannigfaltigkeiten von zwei komplexen Dimensionen. J. reine angew. Math. 191, 110-124 (1953). - [5] HIRzEBRUCH, F.: On Steenrod's reduced powers, the index of inertia, and the Todd genus. Proc. Nat. Acad. Sci. (Wash.) 39, 951-956 (1953). — [6] Hrrzerruch, F.: Neue topologische Methoden in der algebraischen Geometrie. Ergebnisse der Mathematik. Springer-Verlag 1956. - [7] HorF, H.: Zur Topologie der komplexen Mannigfaltigkeiten. Studies and Essays presented to R. Courant, p. 167-185. New York 1948. - [8] HoPf, H.: Sur les champs d'éléments de surface dans les variétés à 4 dimensions. Colloques internat. centre nat. rech. sci. Nr. 12 (Topologie algébrique, Paris 1947), 55-59 (1949), - [9] НоРF, H.: Sur une formule de la théorie des espaces fibrés. Centre Belge rech. math., Coll. Top. 117-121 (1951). - [10] Horf, H.: Die Coinzidenz-Cozyklen und eine Formel aus der Fasertheorie. Algebraic Geometry and Topology. A Symposium in honor of S. LEFschetz. Princeton University Press 1957. - [11] Kund ERT, E. G.: Uber Schnittflächen in speziellen Faserungen und Felder reeller und komplexer Linienelemente. Ann. of Math. 54, 215-246 (1951). - [12] Stmenzod, N. E.: The topology of fibre bundles. Princeton University Press 1951. - [13] Tном, R.: Espaces fibrés en sphères et carrés de Steenrod. Ann. Sci. Ecol. norm. sup. 69, 109-182 (1952). - [14] Tном, R.: Quelques propriétés globales des variétés différentiables. Comm. Math. Helvet. 28, 17 -86 (1954). [15] WhITNeY, H.: On the topology of differentiable manifolds. Lectures in Topology. Ann Arbor, Michigan, 1941. - [16] Wu WEN-Tsun: Classes caractéristiques et $i$-carrés d'une variété. C. R. Acad. Sei. (Paris) 230, 508-511 (1950). - [17] Wu Wen-Tsun: Sur les puissances de Steenrod. Colloque de topologie de Strasbourg 1951 (vervielfältigt).[18] WU WEN-Tsun: Sur les classes caractéristiques des structures fibrées sphériques. Actual. Sei. industr. 1183 (1952). 